\title{
HST-STIS spectroscopy of the Bubble Nebula, NGC 7635
}

\author{
Brent Buckalew ${ }^{1}$, Reginald J. Dufour ${ }^{1}$, Parviz Ghavamian ${ }^{1}$, Patrick \\ Hartigan $^{1}$, Don K. Walter ${ }^{2}$, Jeff J. Hester ${ }^{3}$, and Paul A. Scowen ${ }^{3}$ \\ ${ }^{1}$ Rice University, Houston, TX 77005, USA \\ ${ }^{2}$ South Carolina State University, Orangeburg, SC 29117, USA \\ ${ }^{3}$ Arizona State University, Tempe, AZ 85287-1504, USA
}

\begin{abstract}
We report the results of long-slit spectroscopy of the wind-blown bubble and photo-evaporating knots around the 06.5IIIf star BD $+60^{\circ} 2522$ made with the HST-Space Telescope Imaging Spectrograph. The Of star is the primary ionizing source for the $\mathrm{H}$ II region NGC 7635, located in the Perseus Arm. The spectra were taken through a $0.2 \times 52^{\prime \prime}$ slit with low and medium resolution gratings covering the wavelength range $2900-6870 \AA$. Observations with two slit orientations were made; one across the line of embedded knots to the west of the Of star and the second running from the Of star across the bubble to the NE.

The 2D-STIS spectra permit us to subtract the surrounding H II region's diffuse emission from that of the knots and the bubble, and to study the spatial variations in various emission lines in these features to a resolution of $\sim 0$ !' 2 , an order of magnitude improvement over the best ground-based spectra of this object in the literature. We present high spatial-resolution emission-line and lineratio profiles across the bubble and knots, and compare them with the predicted variations from photo-ionization, photo-evaporation, and wind-shock models. We also present an analysis of temperatures, densities, and abundances in the features from higher $\mathrm{S} / \mathrm{N}$ spectra extracted over selected lengths of the slit.
\end{abstract}

\section{Discussion}

From the spectra shown in Fig. 1, we were able to determine electron densities and temperatures, chemical abundances, and study the spatial variations of certain emission line ratios. We find that our physical parameters agree moderately well with previous results (Talent \& Dufour 1979) and the chemical abundances in the knots and the rim are what we would expect for an $\mathrm{H}$ II region at $12 \mathrm{kpc}$ (Shaver et al. 1983). We have also derived a preliminary $\mathrm{C}^{+} / \mathrm{H}^{+}$ratio of $4.27 \times 10^{-4}$, which is weak evidence for a carbon enriched wind. From our spatial scan studies of the knots and rim, we conclude that the knots are composed of photo-evaporating knots surrounded by an ionization front, confirming the results of the imagery which state that the knots are like the EGGs of M 16 seen face on. The rim appears to be a shell of ionized gas being 'snowplowed' by the wind of the central star. The wind is moving subsonicaly rather than supersonicaly with respect to the giant molecular cloud, confirming previous results from Christopoulou et al. (1995). 


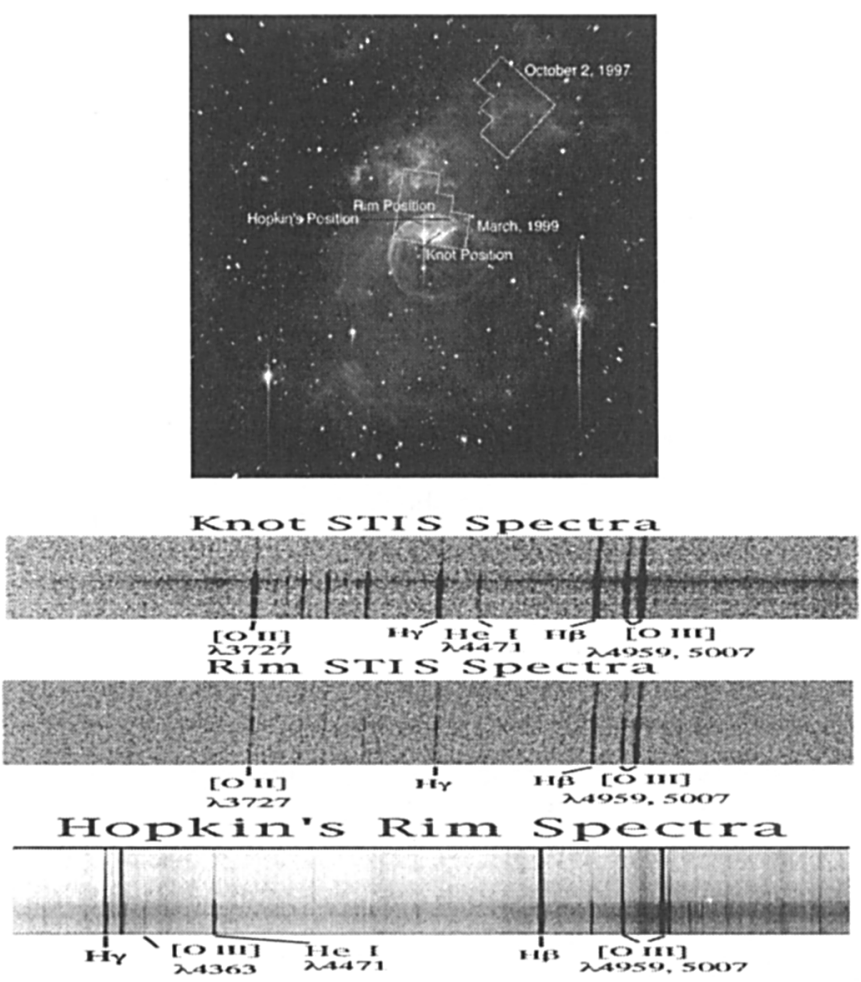

Figure 1. Top: Image of NGC 7635 showing the slit positions taken for this study. The Rim and Knot positions were taken by STIS, and the Hopkin's position was taken at the $1.5 \mathrm{~m}$ at Whipple Observatory. Bottom: The blue spectral portion of each slit position. Important emission lines have been marked.

Acknowledgments. This research was supported in part by grants to ASU, Rice, and SCSU by AURA/STScI related to general observer program GO-7515. B.B. also acknowledges support by NASA-Ames GSRP fellowship NGT 2-52252.

\section{References}

Talent, D.L., Dufour, R.J. 1979, ApJ 233, 888

Shaver, P.A., McGee, R.X., Newton, L.M., Danks, A.C., Pottasch, S.R. 1983, MNRAS 204, 53

Christopoulou, P.E., Goudis, C.D., Meaburn, J., Dyson, J.E., Clayton, C.A. 1995, A\&A 295,509 . 